\title{
LW-NIR hyperspectral imaging for rapid prediction of TVC in chicken flesh
}

\author{
Hui Wang ${ }^{1}$, Hongju He ${ }^{1,2,3^{*}}$, Hanjun Ma ${ }^{1,2}$, Fusheng Chen ${ }^{3}$, Zhuangli Kang ${ }^{1}$, Mingming Zhu ${ }^{1}$, \\ Zhengrong Wang ${ }^{1}$, Shengming Zhao ${ }^{1}$, Rongguang Zhu ${ }^{4}$ \\ (1. School of Food Science, Henan Institute of Science and Technology, Xinxiang 453003, Henan, China; \\ 2. Henan Institute of Science and Technology, Postdoctoral Research Base, Xinxiang 453003, Henan, China; \\ 3. College of Grain, Oil and Food, Henan University of Technology, Zhengzhou 450000, Henan, China; \\ 4. College of Mechanical and Electrical Engineering, Shihezi University, Shihezi 832003, Xinjiang, China)
}

\begin{abstract}
Total viable count (TVC) is often used as an important indicator for chicken freshness evaluation. In this study, 112 fresh chicken flesh samples were acquired after slaughtered and their hyperspectral images were collected in the LW-NIR (900-1700 nm) range. The full LW-NIR spectra (486 wavebands) within the images were extracted and applied to related to reference TVC values measured in different storage periods, using partial least squares regression (PLSR) algorithm, resulting in high correlation coefficients $(R)$ and low root mean square errors (RMSE), for either raw spectra or pretreatment spectra. By using regression coefficients (RC) method, 20,18, 17 and 20 optimal wavebands were respectively selected from raw spectra, baseline correction (BC) spectra, Savitzky-Golay convolution smoothing (SGCS) spectra and standard normal variate (SNV) spectra and applied for the optimization of original full waveband PLSR model. By comparison, RC-PLSR model based on the SGCS spectra showed a better performance in TVC prediction with $R_{\mathrm{C}}$ of 0.98 and RMSEC of $0.35 \log _{10} \mathrm{CFU} / \mathrm{g}$ in calibration set, and $R_{\mathrm{P}}$ of 0.98 and RMSEP of $0.44 \log _{10} \mathrm{CFU} / \mathrm{g}$ in prediction set. At last, by transferring the best RC-PLSR model, the dynamic TVC change during the storage was visualized by color maps to indicate the TVC spoilage degree. The overall study revealed that LW-NIR hyperspectral imaging combined with PLSR could be used to predict the freshness of chicken flesh.
\end{abstract}

Keywords: hyperspectral imaging, chicken, TVC, partial least square regression (PLSR)

DOI: $10.25165 /$ j.ijabe.20191203.4444

Citation: Wang H, He H J, Ma H J, Chen F S, Kang Z L, Zhu M M, et al. LW-NIR hyperspectral imaging for rapid prediction of TVC in chicken flesh. Int J Agric \& Biol Eng, 2019; 12(3): 180-186.

\section{Introduction}

Nowadays, China is the largest country for meat production and consumption, and meat has become one of the must-have foods on the table ${ }^{[1]}$. Meat (pork, beef, lamb, chicken, and others) provides human beings with a lot of valuable protein and other nutritious substances ${ }^{[2,3]}$. Among the different meat products, chicken has more protein and lower fat, which attracts a lot of attention by middle-aged and aged people. Besides, chicken is cheaper and easier to digest and absorb ${ }^{[4]}$. At present, chicken has become the second largest meat products in China.

In general, chicken is purchased and always stored at $0^{\circ} \mathrm{C}-4^{\circ} \mathrm{C}$ for consumption. However, chicken is so easy to be spoiled by

Received date: 2018-05-31 Accepted date: 2018-12-17

Biographies: Hui Wang, Master, research interests: food nondestructive analysis and detection, Email: huiwang169@163.com; Hanjun Ma, Professor, research interest: meat processing and quality control, Email: xxhjma@126.com; Fusheng Chen, Professor, research interests: food resource development and utilization, Email: fushengc@haut.edu.cn; Zhuangli Kang, PhD, research interest: meat processing, Email: kzlnj1988@163.com; Mingming Zhu, PhD, research interest: processing and storage of agricultural products, protein purification, Email: happyzhumingming@126.com; Zhengrong Wang, PhD, research interest: meat quality control and processing, Email:wazhro@qq.com; Shengming Zhao, $\mathrm{PhD}$, research interest: meat safety and control, E-mail:zhaoshengming2008@126.com; Rongguang Zhu, Professor, research interests: food nondestructive analysis and detection, Email: rgzh jd@163.com.

*Corresponding author: Hongju He, Professor, research interest: food nondestructive analysis and detection. Henan Institute of Science and Technology, Postdoctoral Research Base, Xinxiang 453003, China. Tel: +86-373-3040674, +86-13525022325, Fax:+86-373-3040674. Email: hongju_he007@126.com. bacteria and the total viable bacteria count (TVC) value always exceeds the standard value of $6 \log _{10} \mathrm{CFU} / \mathrm{g}$ in $3-5 \mathrm{~d}$ at $4{ }^{\circ} \mathrm{C}$ storage $\mathrm{e}^{[5,6]}$, in this situation, the chicken is not safe for consumption as it would greatly endanger consumers' health. TVC is now often used as an indicator for freshness evaluation and is commonly determined by standard plate colony count ${ }^{[7,8]}$, but this method is time-consuming, laborious, destructive and inefficient. With the continuous development of science and technology, some nondestructive testing methods have been studied in the microbial detection of meat, such as visible/near-infrared spectroscopy ${ }^{[9]}$ and hyperspectral imaging ${ }^{[10]}$. Hyperspectral imaging technology combining spectroscopy and computer vision technology can simultaneously provide the spatial information and spectral information of the tested samples. It has been widely used for the quality evaluation in meat in terms of chemical quality such as fat, protein, moisture ${ }^{[11-13]}$, freshness indexes including total volatile basic nitrogen $(\mathrm{TVB}-\mathrm{N})^{[14]}$, thiobarbituric acid reactive substances $(\mathrm{TBARS})^{[15]}$, biogenic amine index $(\mathrm{BAI})^{[16]}$, and physical properties such as color ${ }^{[17]}$, tenderness ${ }^{[18]}$, springness ${ }^{[19]}$ and water-holding capacity ${ }^{[20]}$.

Besides, hyperspectral imaging has been applied for microbial evaluation in meat. Peng et al. ${ }^{[21]}$ investigated the TVC in beef during every $12 \mathrm{~h}$ storage at $8^{\circ} \mathrm{C}$ by using hyperspectral imaging at 400-1100 nm region, combined with Multiple Linear Regression (MLR) algorithm, and obtained good prediction performance with correlation coefficient $(R)$ of 0.97 and standard error of $0.30 \log _{10} \mathrm{CFU} / \mathrm{g}$. In the same wavelength region, Zheng et al. ${ }^{[22]}$ established a support machine regression (SVR) model to predict the TVC on the pork surface and achieved a better result. Barbin 
et al. ${ }^{[23]}$ investigated the potential of NIR hyperspectral imaging (900-1700 nm) for psychrotrophic plate count (PPC) in chilled pork during 21 days' storage. As for chicken, Ye et al. ${ }^{[24]}$ used the visible and near-infrared (Vis/NIR) hyperspectral imaging (400-1100 nm) to study the TVC value in chicken fillets during $4^{\circ} \mathrm{C}$ storage and the correlation coefficient of only 0.83 was obtained. The results needed to be further improved. These investigations showed that hyperspectral imaging technology has great potential in the quality evaluation of meat. In the 900$1700 \mathrm{~nm}$, however, few reports have been found to evaluate contamination of TVC in chicken by using hyperspectral images. Therefore, the objective of this study was to explore the potential of long-wavelength near-infrared (LW-NIR) hyperspectral imaging for the quantitative determination of TVC in chicken flesh at $4{ }^{\circ} \mathrm{C}$ storage. The spectral data was obtained by LW-NIR hyperspectral imaging system (900-1700 nm), and the optimal waveband associated with TVC in chicken was extracted by chemometrics method. A PLSR prediction model for TVC was established and visualization of TVC distribution was also realized.

\section{Materials and methods}

\subsection{Chicken sample preparation}

One hundred and twelve fresh raw chicken breasts were provided by Henan Zhongpin Food Co., Ltd., China. The length of chicken breasts was $17 \pm 1.5 \mathrm{~cm}$ and the width was $10 \pm 1.5 \mathrm{~cm}$. All samples were vacuum-packed and transported on ice to Meat Processing \& Quality Control Lab, Henan Institute of Science and Technology, Xinxiang, China. A cube with the size of $3 \mathrm{~cm}$ (length) $\times 3 \mathrm{~cm}$ (width) $\times 1 \mathrm{~cm}$ (thickness) was cut from the center part under aseptic condition and a total of 112 subsamples were finally obtained. All the cubed samples were then put into sterile disposable plastic boxes, labeled and stored at $4^{\circ} \mathrm{C}$ for $7 \mathrm{~d}$.

\subsection{Hyperspectral image acquisition and pretreatment}

\subsubsection{Hyperspectral image acquisition}

On each day, 16 samples were taken and scanned by a pushbroom line-scanning hyperspectral reflectance imaging system (Isuzu Optics Corp., Taiwan) which is mainly composed of a spectrograph, a camera, an illumination source, a zoom lens, a moving table, and a computer equipped with an image acquisition software. The moving speed of the platform was $6.33 \mathrm{~mm} / \mathrm{ms}$ the spectrograph exposure time is $4.25 \mathrm{~ms}$ and the distance from the sample to the lens is $45 \mathrm{~cm}$. A total of 112 hyperspectral images of samples were finally obtained.

\subsubsection{Image correction}

The achieved raw hyperspectral images needed to be corrected as the images reflected the light intensity instead of the reflectance information. Besides, the dark current caused by the different intensity of the light source and the noise caused by some factors of the hyperspectral system itself will have adverse effects on the tested samples. In order to eliminate the effects, the obtained raw hyperspectral images can be corrected by the following equation:

$$
R=\frac{R_{0}-R_{\mathrm{d}}}{R_{\mathrm{w}}-R_{\mathrm{d}}}
$$

where, $R$ is corrected hyperspectral image; $R_{0}$ is raw hyperspectral image; $R_{\mathrm{d}}$ is the black image (cover the camera lens completely to get the image, the reflectance is about $0 \%$ ); $R_{\mathrm{w}}$ is the white image (the image was obtained by scanning a whiteboard, the reflectance is about $99.9 \%$ ).

2.2.3 Spectral data extraction and pretreatment

After the image correction, a square shape $(3 \mathrm{~cm} \times 3 \mathrm{~cm})$ in the hyperspectral image was selected as the region of interest (ROI), and the spectral data within the ROI were extracted by using software HSI Analyzer (Isuzu Optics Corp., Taiwan) and then the average spectral data of each sample was calculated and achieved. Finally, 112 mean spectra corresponding to the 112 samples were obtained. The mean spectra of all samples were saved in a matrix where the rows represented the 112 samples and the columns represented the 486 wavebands, the spectroscopic resolution was $1.6 \mathrm{~nm}$.

During the spectral acquisition, spectral is influenced by the noise produced from the instrument and the external environment. Therefore, it is necessary to implement the spectral pretreatment in order to eliminate these interferences. In this study, the obtained raw spectra were pretreated by Savitzky-Golay convolution smoothing (SGCS), baseline correction (BC), and standard normal variate (SNV), respectively. The SGCS is one of the common methods used to eliminate random noise and improve the signal-to-noise ratio ${ }^{[25,26]} . \quad \mathrm{BC}$ is another method firstly proposed by Pearson and Walter to eliminate noise, based on decomposition, reconstruction, and low-frequency wavelet coefficients ${ }^{[27,28]}$. SNV aims to remove the additive baseline and the multiplicative signal effects which are caused by light scattering and to improve the accuracy of spectral data ${ }^{[29,30]}$.

\subsection{TVC determination}

TVC on chicken sample surface was measured by using the standard plate colony counting method ${ }^{[23]}$. In specific, after image acquisition, every sample was immediately put into a sterile blender bag (Stomacher, London, UK) and homogenized with $90 \mathrm{~mL}$ buffered peptone water $(0.1 \%$ peptone $+0.85 \% \mathrm{NaCl})$. Decimal dilutions were then obtained by adding $1 \mathrm{~mL}$ bacteria suspension into $9 \mathrm{~mL}$ buffered peptone water. Subsequently, $0.1 \mathrm{~mL}$ bacteria suspension was quickly taken and added into plate count agar (CM0325, Oxoid, UK), and then spread bacteria solution evenly with sterile inoculation rods. Finally, the Petri dishes were inverted and incubated at $35^{\circ} \mathrm{C}$ for $48 \mathrm{~h}$. After cultivation, the colony count of each Petri dish between 30 and 300 was recorded, calculated and transformed into common ten-based logarithm values $\left(\log _{10} \mathrm{CFU} / \mathrm{g}\right)$.

\subsection{Relationship establishment between spectra and TVC}

The quantitative relationship between the extracted reflectance data and the TVC values was established by using partial least square regression (PLSR) to predict the chicken freshness. PLSR is always used for modeling when the number of wavebands is larger than that of reference values (e.g. TVC) and meanwhile there is high collinearity among the wavebands and reference values. In this study, the spectral information in the range of 900-1700 nm was mined to relate to TVC values. The established PLSR model was evaluated using correlation coefficient of calibration $\left(R_{\mathrm{c}}\right)$ and prediction $\left(R_{\mathrm{p}}\right)$ and root mean square error of calibration (RMSEC) and prediction (RMSEP).

\subsection{Optimal waveband selection and model optimization}

Optimal wavebands are necessary to select for PLSR model optimization, as hundreds of spectra often have redundant information and multicollinearity among contiguous wavebands, which reduces efficiency for data processing ${ }^{[31]}$. Besides, irrelevant information among wavebands will weaken the model performance. Therefore, it is important and necessary to conduct the waveband selection to retain the wavebands holding the most useful information and eliminate the useless wavebands, which will reduce the spectral information redundancy and multicollinearity, and also accelerate the data processing and modeling. In this study, regression coefficients (RC) of PLSR model was applied for 
optimal wavebands selection. In this method, the wavebands corresponding to large values of RC (regardless of sign) are considered to carry the most important spectral information and should be recommended to be selected ${ }^{[32]}$. Wavebands selection by RC method was implemented using software Unscrambler 9.7 (CAMO, Oslo, Norway).

\subsection{TVC visualisation}

Visualisation is an advantage of hyperspectral imaging and it enable hyperspectral images to offer the spatial distribution of quality attributes in the tested samples. In other words, quality attributes will be visualized in a visual image (distribution map). Generally, in this study, the visualization process was realized by two steps. Firstly, the established PLSR models based on the optimal wavebands will be evaluated in terms of $R$ and RMSE values, and the optimized PLSR model with best performance will be selected. Secondly, the optimized PLSR model will be transferred into each pixel of hyperspectral images by using image processing algorithm, and the final colour distribution map will be generated to show the different TVC values in each pixel. The visualisation procedure was implemented by Matlab R2010b software (The Mathworks, Inc., Natick, MA, USA).

\section{Results and discussion}

\subsection{Reference TVC values}

The results of TVC values for the 112 chicken samples are
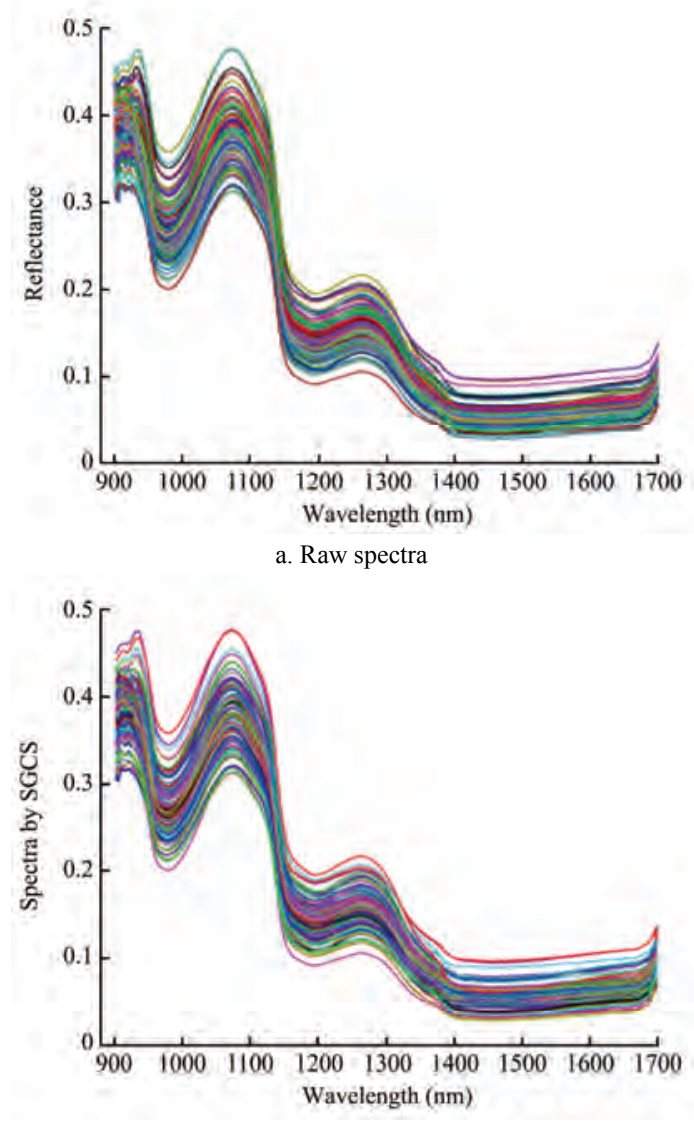

c. SGCS pretreatment spectra shown in Table 1. Among the 112 samples, 84 samples were used for the model calibration and the remaining 28 samples for prediction.

Table 1 TVC values $\left(\log _{10} \mathrm{CFU} / \mathrm{g}\right)$ measured by standard PCA method

\begin{tabular}{ccccc}
\hline Sample set & No. of samples & Minimum & Maximum & Mean \pm SD \\
\hline Calibration & 84 & 3.040 & 9.518 & $5.693 \pm 1.679$ \\
Prediction & 28 & 3.146 & 9.322 & $5.757 \pm 1.832$ \\
\hline
\end{tabular}

Note: SD: standard deviation.

\subsection{Spectral profiles of chicken samples}

The average spectra extracted from the chicken samples in the wavelength range of 900-1700 $\mathrm{nm}$ are shown in Figure 1. Figure 1a showed raw spectra of chicken samples, Figures 1b-1d showed the three different pretreatment spectra of chicken samples. In the four plots, 112 mean spectra had the same trends, and several apparent absorption peaks emerged in the 900-1700 nm region. Two strong absorption peaks at about $980 \mathrm{~nm}$ and $1200 \mathrm{~nm}$ were observed and attributed to the $\mathrm{O}-\mathrm{H}$ stretching (water) and $\mathrm{C}-\mathrm{H}$ stretching (fat), respectively. Another water absorption peak at about 1450 related to $\mathrm{O}-\mathrm{H}$ stretching was not obvious. Protein absorption peak was not found as it was immersed in water information, resulting from the high content of water in chicken flesh.
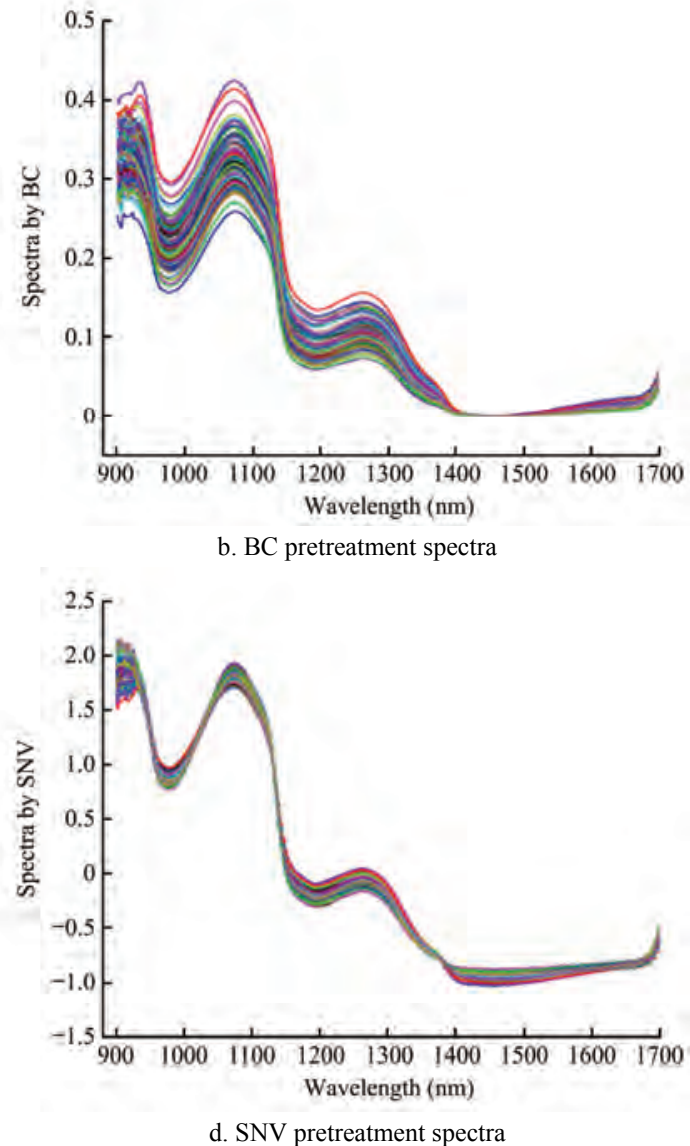

d. SNV pretreatment spectra

\subsection{TVC prediction using full $L W-N I R$ wavelength}

By inputting the full 486 wavebands, a matrix with 112 samples $\times 486$ wavebands was arranged for PLSR model establishment. Among, the sub-matrix with 84 samples $\times 486$ wavebands were used for PLSR calibration and the rest sub-matrix with 28 samples $\times 486$ wavebands were used for PLSR model validation. The established PLSR models based on the full raw, BC, SGCS and SNV pretreatment LW-NIR spectra are shown in Table 2. The four different PLSR models exhibited a similar good performance in TVC prediction, with both the $R_{\mathrm{C}}$ and $R_{\mathrm{P}}$ values being close to 1.00 . The small |RMSEC-RMSEP $\mid$ values also indicated good robustness of the PLSR models. 
Table 2 TVC prediction by PLSR model based on the full LW-NIR wavebands

\begin{tabular}{|c|c|c|c|c|c|c|c|}
\hline \multirow{2}{*}{ Spectra } & \multirow{2}{*}{ Model } & \multirow{2}{*}{$\begin{array}{l}\text { Number of } \\
\text { wavebands }\end{array}$} & \multicolumn{2}{|c|}{ Calibration set } & \multicolumn{2}{|c|}{ Prediction set } & \multirow{2}{*}{$\mid$ RMSEC-RMSEP $\mid$} \\
\hline & & & $R_{\mathrm{c}}$ & RMSEC & $R_{\mathrm{P}}$ & RMSEP & \\
\hline Raw & PLSR & 486 & 0.98 & 0.30 & 0.98 & 0.42 & 0.12 \\
\hline $\mathrm{BC}$ & PLSR & 486 & 0.98 & 0.35 & 0.97 & 0.46 & 0.11 \\
\hline SGCS & PLSR & 486 & 0.98 & 0.35 & 0.98 & 0.44 & 0.09 \\
\hline SNV & PLSR & 486 & 0.96 & 0.48 & 0.94 & 0.64 & 0.16 \\
\hline
\end{tabular}

Note: The units of RMSEC and RMSEP are both $\log 10 \mathrm{CFU} / \mathrm{g}$.

\subsection{Selection of optimal wavebands}

Using the RC method, optimal wavebands from the raw, BC, SGCS and SNV spectra were respectively selected and are shown in Figure 2. As indicated, 20 (Figure 2a), 18 (Figure 2b), 17 (Figure 2c) and 20 (Figure 2d) optimal wavebands were respectively selected from the 900-1700 nm range. Compared with the full 486 wavebands, $95.8 \%, 96.3 \%, 96.5 \%$ and $95.8 \%$ wavebands reduced from the full range, for raw, BC, SGCS and SNV pretreatment spectra, respectively.
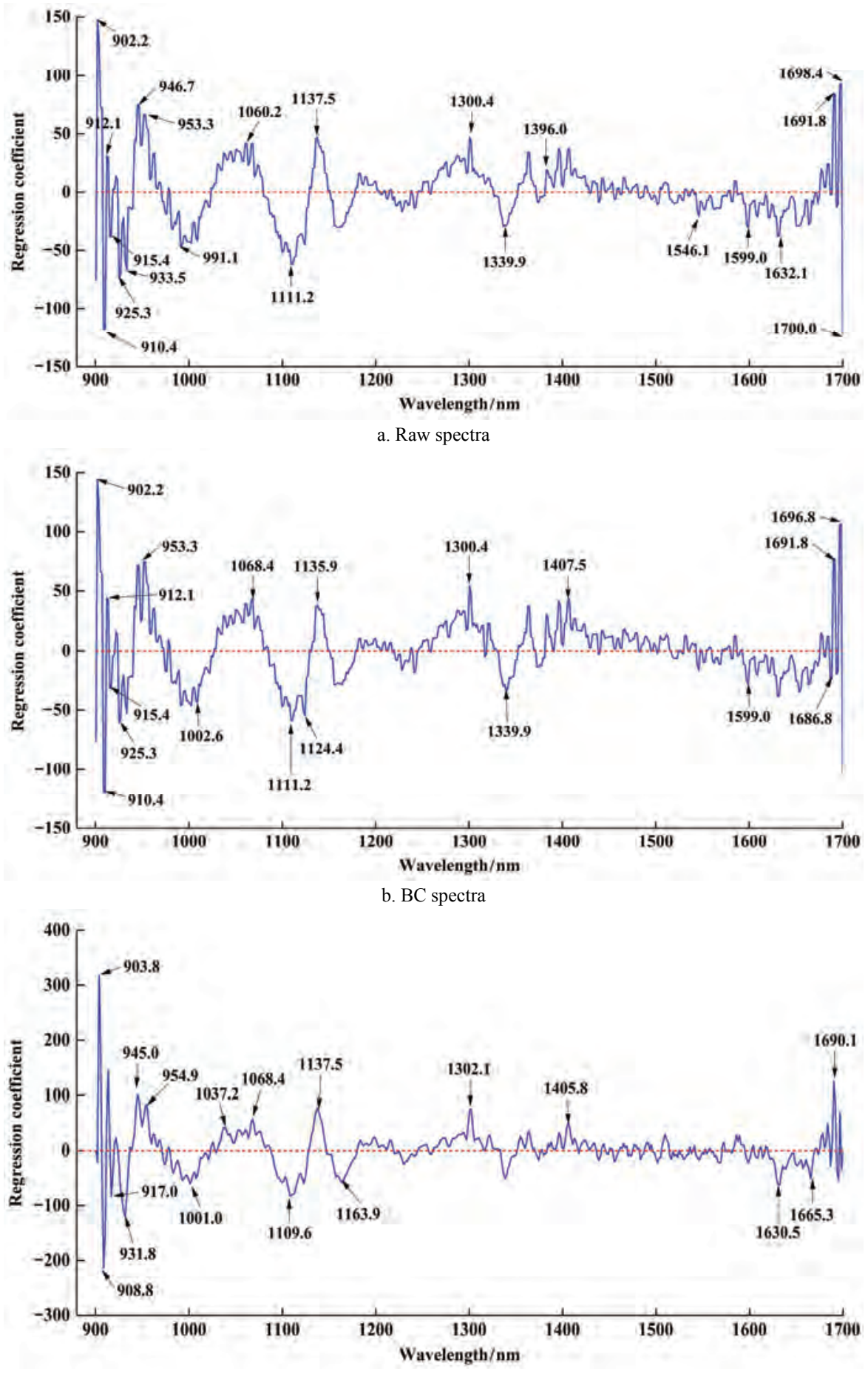

c. SGCS spectra 


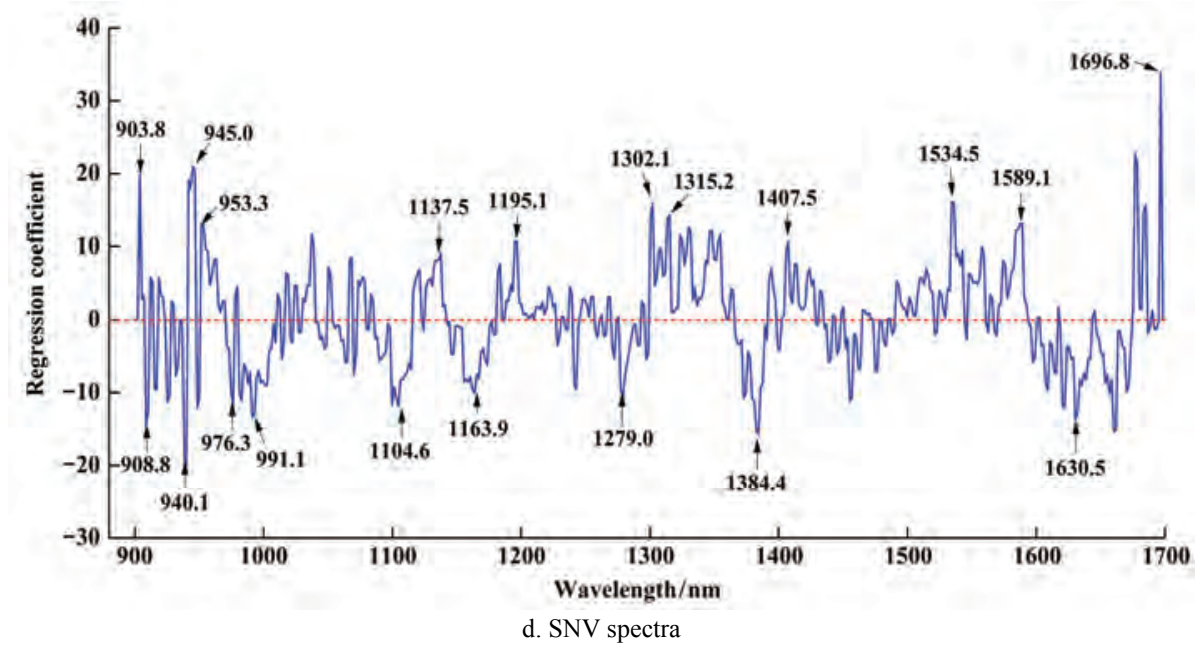

Figure 2 Optimal wavebands selected from raw spectra, BC spectra, SGCS spectra and SNV spectra in the wavelength of 900-1700 nm

\subsection{TVC prediction based on optimal wavebands}

Based on the selected optimal wavebands, new matrix with sample and optimal wavebands were built for model optimization. With 20 optimal wavebands selected from raw LW-NIR spectra, 84 samples $\times 20$ wavebands were input for PLSR model calibration and the rest 28 samples $\times 20$ wavebands were input for PLSR model validation. Similarly, PLSR model optimization based on the optimal wavebands selected from BC, SGSC and SNV spectra were also conduced and all results are shown in Table 3 .

As indicated, the four optimized PLSR models performed well in TVC prediction, with high correlation coefficients and low RMSE values. Besides, the low |RMSEC-RMSEP $\mid$ values also showed the good robustness of the four optimized PLSR models. Among, the PLSR model based on the optimal waveband selected from SGCS spectra showed a little better performance in TVC prediction than the other three optimize PLSR models, and also showed the least wavebands number (17) among the four optimized PLSR models. Contrasted with PLSR models from the full waveband spectra, the performance of the RC-PLS model using SGCS spectra was similar to that of PLSR models using the full waveband spectra for TVC prediction. It was found that the number of wavelengths was reduced from 486 to 17 , which means that the computer calculation speed will be improved and higher efficiency for the TVC prediction using selective optimal wavebands will be achieved in the future. Moreover, the $\mid$ RMSEC-RMSEP $\mid$ value in RC-PLSR model was $0.02,78 \%$ less than that of PLSR model from the full waveband spectra, which showed that the RC-PLSR model had better robustness than the PLSR model. According to the regression coefficients of the RC-PLS model from SGCS spectra, a formula for the prediction of TVC loads of chicken flesh was obtained and is shown below:

$Y_{\mathrm{TVC}}=30.762+317.544 X_{903.8 \mathrm{~nm}}-213.711 X_{908.8 \mathrm{~nm}}-83.41 X_{917.0 \mathrm{~nm}}-$ $120.315 X_{931.8 \mathrm{~nm}}+100.056 X_{945.0 \mathrm{~nm}}+83.501 X_{954.9 \mathrm{~nm}}-60.374 X_{1001.0 \mathrm{~nm}}$ $+42.225 X_{1037.2 \mathrm{~nm}}+56.921 X_{1068.4 \mathrm{~nm}}-81.112 X_{1109.6 \mathrm{~nm}}+76.338 X_{1137.5 \mathrm{~nm}}$

$-57.781 X_{1163.9 \mathrm{~nm}}+75.853 X_{1302.0 \mathrm{~nm}}+51.835 X_{1405.8 \mathrm{~nm}}-60.909 X_{1630.5 \mathrm{~nm}}$ $-51.644 X_{1665.3 \mathrm{~nm}}+125.476 X_{1690.1 \mathrm{~nm}}$

Table 3 TVC prediction by RC-PLSR model based on the selected optimal wavebands

\begin{tabular}{|c|c|c|c|c|c|c|c|}
\hline \multirow{2}{*}{ Spectra } & \multirow{2}{*}{ Model } & \multirow{2}{*}{$\begin{array}{l}\text { Number of } \\
\text { waveband }\end{array}$} & \multicolumn{2}{|c|}{ Calibration set } & \multicolumn{2}{|c|}{ Prediction set } & \multirow{2}{*}{$\mid$ RMSEC-RMSEP $\mid$} \\
\hline & & & $R_{\mathrm{c}}$ & RMSEC & $R_{\mathrm{P}}$ & RMSEP & \\
\hline Raw & RC-PLSR & 20 & 0.96 & 0.47 & 0.96 & 0.56 & 0.09 \\
\hline $\mathrm{BC}$ & RC-PLSR & 18 & 0.95 & 0.51 & 0.93 & 0.66 & 0.15 \\
\hline SGCS & RC-PLSR & 17 & 0.96 & 0.46 & 0.98 & 0.44 & 0.02 \\
\hline SNV & RC-PLSR & 20 & 0.94 & 0.58 & 0.89 & 0.84 & 0.26 \\
\hline
\end{tabular}

Note: The units of RMSEC and RMSEP are both $\log 10 \mathrm{CFU} / \mathrm{g}$.

\subsection{TVC distribution map}

Besides providing the spectra for PLSR prediction model establishment, hyperspectral imaging offers visualization function for visualizing the TVC distribution. In this study, the TVC visualization was conducted by transferring the RC-PLSR model to each pixel of hyperspectral images. In specific, the spectrum of each pixel within the chicken fillet was first reduced to 17 optimal wavebands selected by RC, and 17 optimal wavebands were set as the inputs of the RC-PLSR model using SGCS spectra and the TVC value of the pixel was set as the output value. When the TVC values of all pixels in each ROI were calculated, the TVC distribution map was generated based on the spatial positions of each ROI. Finally, distribution maps were displayed according to a linear colour scale and shown in Figure 3. In these maps, all pixels having similar spectral features produced the similar predicted TVC values and visualized in the similar colour. As shown in Figure 3, a general chicken spoilage trend by TVC was observed with the mean TVC values increase from 3.141 to 9.313 . At early storage time, blue colour occupied the most in the distribution map, which means the chicken spoilage by TVC was not serious. At the middle stage, green and red colour was observed to be the most colour in the map, which indicated the serious TVC spoilage in the chicken. At the later period, red color accounted for the majority of the colour, demonstrating the very serious TVC spoilage in chicken flesh. The distribution maps combined with the RC-PLSR model indicated that LW-NIR hyperspectral imaging could be a useful and effective technology in predicting TVC spoilage of chicken flesh. 


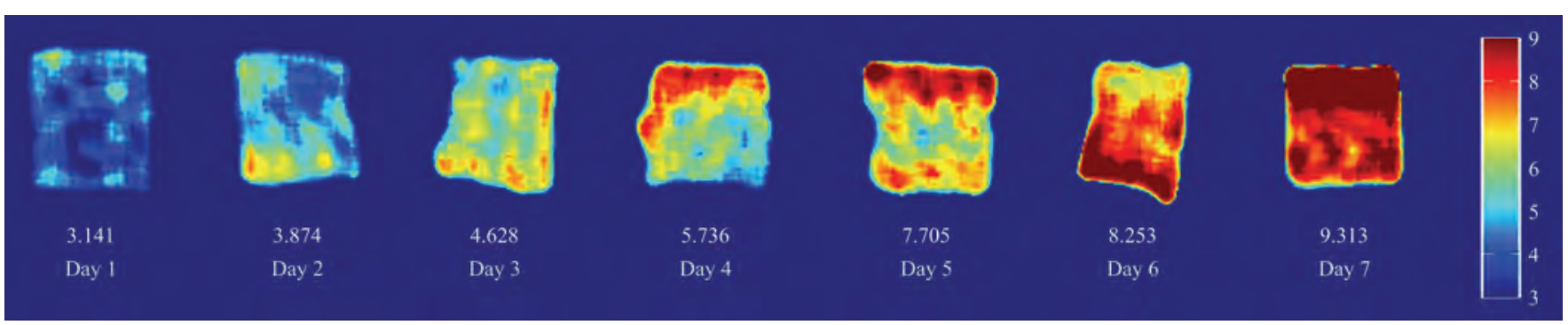

Figure 3 TVC distribution map of some chicken samples at different storage period

\section{Conclusions}

In this work, the application of LW-NIR hyperspectral imaging (900-1700 nm) for TVC prediction in chicken flesh was conducted. The hyperspectral images of chicken samples containing spectral and spatial information were obtained at different storage periods. The spectra were extracted and related to reference TVC values by PLSR, resulting in the high $R$ and low RMSE, either for calibration $\left(R_{\mathrm{C}} \& \mathrm{RMSEC}\right)$ or for prediction $\left(R_{\mathrm{P}} \& \mathrm{RMSEP}\right)$. Through $\mathrm{RC}$ method, 20, 18, 17 and 20 optimal wavebands were selected from raw spectra, BC spectra, SGCS spectra and SNV spectra. Based on these optimal wavebands, the original PLSR models were optimized and kept similar good performance in TVC predication of chicken flesh. Among, the PLSR model based on the 17 optimal wavebands $(903.845,908.787,917.022,931.844,945.017$, 954.896, 1000.985, 1037.187, 1068.446, 1109.572, 1137.536, $1163.856,132.073,1405.841,1630.464,1665.252$ and $1690.121 \mathrm{~nm})$ showed a better performance $\left(R_{\mathrm{P}}=0.98, \mathrm{RMSEP}=0.44\right)$ and was finally transferred for generating TVC distribution maps. The overall results indicated that hyperspectral imaging ranging from 900-1700 $\mathrm{nm}$ has a great potential and could be used as a replacement for traditional techniques in TVC evaluation of chicken flesh.

\section{Acknowledgements}

The authors would like to acknowledge the financial support provided by Major Scientific and Technological Project of Henan Province (No. 161100110600), China Postdoctoral Science Foundation (No. 2018M632767), Key Scientific and Technological Project of Henan Province (No. 182102310060, No. 182102110091), Youth Talents Lifting Project of Henan Province (No. [2017]132-08), Key Scientific Research Project of Henan Province (No. 18A550007) and National Natural Science Foundation of China (No. 31860465).

\section{[References]}

[1] Chen Y N, Sun D W, Cheng J H, Gao W H. Recent advances for rapid identification of chemical information of muscle foods by hyperspectral imaging analysis. Food Engineering Reviews, 2016; 8(3): 336-350.

[2] Kamruzzaman M, Elmasry G, Sun D W, Allen P. Non-destructive prediction and visualization of chemical composition in lamb meat using NIR hyperspectral imaging and multivariate regression. Innovative Food Science \& Emerging Technologies, 2012; 16(39): 218-226.

[3] Wu X, Song X, Qiu Z, He Y. Mapping of TBARS distribution in frozen-thawed pork using NIR hyperspectral imaging. Meat Science, 2016; 113: 92-96.

[4] Berzaghi P, Dalle Z A, Jansson L M, Andrighetto I. Near-infrared reflectance spectroscopy as a method to predict chemical composition of breast meat and discriminate between different n-3 feeding sources. Poultry Science, 2005; 84(1): 128-136.

[5] Ntzimani A G, Giatrakou V I, Savvaidis I N. Combined natural antimicrobial treatments (EDTA, lysozyme, rosemary and oregano oil) on semi cooked coated chicken meat stored in vacuum packages at $4^{\circ} \mathrm{C}$ :
Microbiological and sensory evaluation. Innovative Food Science \& Emerging Technologies, 2010; 11(1): 187-196.

[6] He H J, Sun D W. Hyperspectral imaging technology for rapid detection of various microbial contaminants in agricultural and food products. Trends in Food Science \& Technology, 2015; 46(1): 99-109.

[7] Jongenburger I, Reij M W, Boer E P, Gorris L G, Zwietering M H. Factors influencing the accuracy of the plating method used to enumerate low numbers of viable micro-organisms in food. International Journal of Food Microbiology, 2010; 143(1-2): 32-40.

[8] Sieuwerts S, De Bok F A M D, Mols E, Vos W M D, Vlieg J E T V H. A simple and fast method for determining colony forming units. Letters in Applied Microbiology, 2008; 47(4): 275-278.

[9] Xiccato G, Trocino A, Tulli F, Tibaldi E. Prediction of chemical composition and origin identification of European sea bass (Dicentrarchus labrax, L.) by near infrared reflectance spectroscopy (NIRS). Food Chemistry, 2004; 86(2): 275-281.

[10] Wu D, Sun D W, He Y. Application of long-wave near infrared hyperspectral imaging for measurement of color distribution in salmon fillet. Innovative Food Science \& Emerging Technologies, 2012; 16(39): 361-372.

[11] ElMasry G, Sun D W, Allen P. Chemical-free assessment and mapping of major constituents in beef using hyperspectral imaging. Journal of Food Engineering, 2013; 117(2): 235-246.

[12] Barbin D F, ElMasry G, Sun D W, Allen P. Non-destructive determination of chemical composition in intact and minced pork using near-infrared hyperspectral imaging. Food Chemistry, 2013; 138(2-3): 1162-1171.

[13] Pu H, Sun D W, Ma J, Liu D, Kamruzzaman M. Hierarchical variable selection for predicting chemical constituents in lamb meats using hyperspectral imaging. Journal of Food Engineering, 2014; 143(2): 44-52.

[14] Khulal U, Zhao J, Hu W, Chen Q. Nondestructive quantifying total volatile basic nitrogen (TVB-N) content in chicken using hyperspectral imaging (HSI) technique combined with different data dimension reduction algorithms. Food Chemistry, 2016; 197: 1191-1199.

[15] Xiong Z, Sun D W, Pu H, Xie A, Han Z, Luo M. Non-destructive prediction of thiobarbituric acid reactive substances (TBARS) value for freshness evaluation of chicken meat using hyperspectral imaging. Food Chemistry, 2015; 179(1): 175-181.

[16] Cheng W, Sun D W, Cheng J H. Pork biogenic amine index (BAI) determination based on chemometric analysis of hyperspectral imaging data. LWT-Food Science and Technology, 2016; 73: 13-19.

[17] ElMasry G, Sun D W, Allen P. Near-infrared hyperspectral imaging for predicting colour, $\mathrm{pH}$ and tenderness of fresh beef. Journal of Food Engineering, 2012; 110(1): 127-140.

[18] He H J, Wu D, Sun D W. Potential of hyperspectral imaging combined with chemometric analysis for assessing and visualising tenderness distribution in raw farmed salmon fillets. Journal of Food Engineering, 2014; 126(1): 156-164.

[19] Xiong Z, Sun D W, Dai Q, Han Z, Zeng X A, Wang L. Application of visible hyperspectral imaging for prediction of springiness of fresh chicken meat. Food Analytical Methods, 2015; 8(2): 380-391.

[20] ElMasry G, Sun D W, Allen P. Non-destructive determination of water-holding capacity in fresh beef by using NIR hyperspectral imaging. Food Research International, 2011; 44(9): 2624-2633.

[21] Peng Y K, Tao F F, Li Y Y, Wang W, Chen J J, Wu J H, et al. Rapid detection of total viable count of chilled pork using hyperspectral scattering technique. Proceedings of SPIE, April 6-7, 2010; Paper No. 7676-21.

[22] Zheng X, Peng Y, Wang W. A nondestructive real-time detection method of total viable count in pork by hyperspectral imaging technique. Applied Sciences, 2017; 7(3): 213-221.

[23] Barbin D F, ElMasry G, Sun D W, Allen P, Morsy N. Non-destructive assessment of microbial contamination in porcine meat using NIR 
hyperspectral imaging. Innovative Food Science \& Emerging Technologies, 2013; 17(17): 180-191.

[24] Ye X, Iino K, Zhang S. Monitoring of bacterial contamination on chicken meat surface using a novel narrowband spectral index derived from hyperspectral imagery data. Meat Science, 2016; 122: 25-31.

[25] Savitzky A, Golay M J E. Smoothing and differentiation of data by simplified least squares procedures. Analytical Chemistry, 1964; 36(8): 1627-1639.

[26] Dai Q, Sun D, Xiong Z, Cheng J, Zeng X. Recent advances in data mining techniques and their applications in hyperspectral image processing for the food industry. Comprehensive Reviews in Food Science \& Food Safety, 2014; 13(5): 891-905.

[27] Sayadi O, Shamsollahi M B. Multiadaptive bionic wavelet transform: application to ECG denoising and baseline wandering reduction. Eurasip Journal on Advances in Signal Processing, 2007; 2007(1): 41274-41284.

[28] Tan H W, Brown S D. Wavelet analysis applied to removing non - constant, varying spectroscopic background in multivariate calibration. Journal of Chemometrics, 2002; 16(5): 228-240.

[29] Kandpal L M, Lee H, Kim M S, Mo C, Cho B K. Hyperspectral reflectance imaging technique for visualization of moisture distribution in cooked chicken breast. Sensors, 2013; 13(10): 13289-13300.

[30] Kamruzzaman M, Makino Y, Oshita S. Rapid and non-destructive detection of chicken adulteration in minced beef using visible near-infrared hyperspectral imaging and machine learning. Journal of Food Engineering, 2016; 170(7): 8-15.

[31] He H J, Sun D W. Selection of informative spectral wavelength for evaluating and visualising Enterobacteriaceae contamination of salmon flesh. Food Analytical Methods, 2015; 8(10): 2427-2436.

[32] He H J, Wu D, Sun D W. Non-destructive and rapid analysis of moisture distribution in farmed Atlantic salmon (Salmo salar) fillets using visible and near-infrared hyperspectral imaging. Innovative Food Science \& Emerging Technologies, 2013; 18(2): 237-245. 